\title{
COMPUESTOS BIOACTIVOS Y CAPACIDAD ANTIOXIDANTE EN FRUTOS DE XOCOTUNA, TUNA Y XOCONOSTLE (Opuntia spp.)
}

\author{
BIOACTIVE COMPOUNDS AND ANTIOXIDANT CAPACITY IN FRUITS \\ OF XOCOTUNA, CACTUS PEAR AND XOCONOSTLE (Opuntia spp.)
}

\author{
Teresa Monroy-Gutiérrez ${ }^{1}$, Ma. Teresa Martínez-Damián ${ }^{1 *}$, Alejandro. F. Barrientos-Priego ${ }^{1}$, \\ Clemente Gallegos-Vázquez ${ }^{2}$, Oscar Cruz-Alvarez ${ }^{3}$, Haidel Vargas-Madríz ${ }^{1}$
}

${ }^{1}$ Departamento de Fitotecnia, Universidad Autónoma Chapingo, Carretera México-Texcoco, Km. 38.5, CP 56230, Estado de México, México.

${ }^{2}$ Centro Universitario Centro Norte (CRUCEN), Universidad Autónoma Chapingo, Cruz del Sur Núm. 100, Constelaciones, Zacatecas, CP. 98060 . Zacatecas, México.

${ }^{3}$ Facultad de Ciencias Agrotecnológicas, Universidad Autónoma de Chihuahua, Avenida Pascual Orozco s/n, Campus 1, Santo Niño, CP 31350, Chihuahua, México.

Autor de correspondencia E-mail: teremd13@gmail.com

\section{RESUMEN}

El objetivo de la presente investigación fue determinar el contenido de algunos compuestos bioactivos y la capacidad antioxidante de xocotuna (Opuntia chiangiana) en comparación con tuna (Opuntia ficus-indica) y xoconostle (Opuntia spp.). Se cosecharon frutos en madurez fisiológica durante los meses de junio, agosto y octubre de 2013 en el municipio de El Orito, Zacatecas, México. Las variables evaluadas fueron: capacidad antioxidante, fenoles totales, vitamina $C$, contenido de betalaínas, clorofila total y carotenos. Se observó que el cultivar de xocotuna 'Coral' presentó diferencias estadísticas a los 3, 6, 9 y 12 días de almacenamiento con los niveles más altos de contenido de capacidad antioxidante (entre 0,92 y 1,04 mg VCEAC $\mathrm{g}^{-1}$ ). El contenido de fenoles totales fue significativamente mayor en 'Cuaresmeño' con valores iniciales de 44,61 mg EAG $100 \mathrm{~g}^{-1}$, mientras que 'Amarilla Plátano' tuvo el menor valor con 6,27 mg EAG $100 \mathrm{~g}^{-1}$. Se encontraron diferencias significativas en el contenido de vitamina $C$ donde los cultivares de xoconostle mostraron los niveles más altos durante el periodo evaluado (entre 41,80 y 125,98 mg $100 \mathrm{~g}^{-1}$ ). Con relación al contenido de betalaínas, clorofila total y carotenos, se encontró una alta variabilidad debido al color característico de cada cultivar. Los cultivares analizados, principalmente los de xoconostle y xocotuna, podrían ser considerados una excelente fuente alternativa de vitamina $\mathrm{C}$ y antioxidantes para ser incorporados a la dieta o como ingredientes naturales en la elaboración de otros productos.

Palabras clave: xocotuna, tuna, xoconostle, Opuntia spp., cactus, carotenos, ácido ascórbico, betalaína.

\section{ABSTRACT}

The objective of this research was to determine the content of some bioactive compounds and the antioxidant potential of xocotuna (Opuntia chiangiana) compared to that of cactus pear (Opuntia ficus-indica) and xoconostle (Opuntia spp.). Fruits at physiological maturity were harvested in the months of June, August and October of 2013 in El Orito, Zacatecas, México. The variables evaluated were: antioxidant capacity, total phenolic content, vitamin $\mathrm{C}$, betalain content, and total chlorophyll and carotene contents. Statistical differences were observed in 'Coral' xocotuna after 3, 6, 9 and 12 days of storage, reaching the highest levels of antioxidant capacity (between 0.92 and $1.04 \mathrm{mg}$ VCEAC

Recibido: 27 julio 2017. Aceptado: 24 agosto 2017. 
$\left.\mathrm{g}^{-1}\right)$. 'Cuaresmeño' recorded the highest total phenolic content with initial values of $44.61 \mathrm{mg}$ GAE 100 $\mathrm{g}^{-1}$, while 'Amarilla Plátano' recorded the lowest value of $6.27 \mathrm{mg} \mathrm{GAE} 100 \mathrm{~g}^{-1}$. Significant differences were also found in the content of vitamin $C_{\text {; }}$ xoconostle cultivars showed the highest levels during

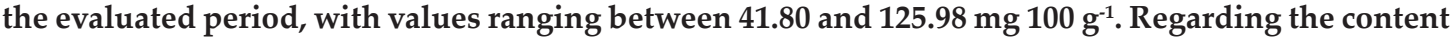
of betalain, and total chlorophyll and carotene contents, a high variability was found because of the characteristic color of each cultivar. The cultivars analyzed, mainly those of xoconostle and xocotuna, could be considered as an excellent source of vitamin $C$ and antioxidants to be included in the diet or as natural ingredients to prepare other products.

Key words: xocotuna, tuna, xoconostle, Opuntia spp., prickly pear, carotene, ascorbic acid, betalain.

\section{INTRODUCCIÓN}

Las cactáceas son una familia de plantas que en México se encuentran ampliamente representadas, con una gran diversidad en el país y una amplia gama de usos, donde existen más de 40 especies entre silvestres y domesticadas. Bajo este contexto, Opuntia es un género complejo que incluye especies usadas para el consumo de cladodios tiernos, conocidos como "nopalitos", obtenidos principalmente de O. ficus-indica, o por sus frutos, llamados tunas y xoconostles (Bravo y Scheinvar, 1999; Gallegos-Vázquez et al., 2011).

Dentro del género Opuntia, la mayoría de las especies producen frutos de sabor dulce (tunas), y el resto produce frutos de sabor ácido, los cuales son conocidos como xoconostles. En general, los frutos poseen altos contenidos de $\mathrm{Ca}$, $\mathrm{K}$, azúcares, fibra dietética, ácido ascórbico, polifenoles, carotenoides y pigmentos como betacianinas, los cuales han sido relacionados con algunos beneficios para la salud, entre estos, el de ser compuestos con una potente capacidad antioxidante, por lo que podrían ser atractivos para la industria alimenticia (Saénz, 2006; Pimienta-Barrios et al., 2008; PrietoGarcía et al., 2008; Osorio-Esquivel et al., 2011).

El género Opuntia es muy importante para nuestro país como recurso fitogenético como consecuencia de la amplia variabilidad existente y a las propiedades nutricionales y funcionales que posee (Reyes-Agüero et al., 2005). En este aspecto Scheinvar y Manzanero (2009) han descrito una nueva especie de Opuntia (L.) Miller (Cactaceae) denominada por los agricultores locales como xocotuna (Opuntia chiangiana), indicando que se trata de una forma intermedia entre tuna y xoconostle, la cual se caracteriza por tener las paredes de los frutos y los funículos semisecos, de sabor agridulce, que cuando maduran, permanecen adheridos a los cladodios por más de un año, tal como ocurre con los xoconostles. Aunque las xocotunas son un recurso fitogenético con un enorme potencial, no existen estudios que las caractericen como alimento funcional, por tanto, el objetivo fue estudiar el contenido de algunos compuestos bioactivos y la capacidad antioxidante con relación a la tuna y xoconostle.

\section{MATERIALES Y MÉTODOS}

\section{Material vegetal}

Se utilizaron frutos de cinco cultivares de xocotuna: 'Cascarón' (O. chavena Griffiths), 'Chinchilla' (O. streptacantha Lem.), 'Coral' (O. affinis lindheimeri), 'Nicolaita' y 'Caidilla' (Opuntia sp.), seis cultivares de tuna: 'Amarilla Montesa' y 'Amarilla Plátano' (O. megacantha SalmDick), 'Reyna' y 'Cristalina' (Opuntia albicarpa Scheinvar), 'Rojo Pelón' y 'Liso Forrajero' (Opuntia ficus-indica (L.) Mill.); y dos cultivares de xoconostle: 'Cuaresmeño Blanco' y 'Cuaresmeño' (O. matudae Scheinvar). Los frutos se cosecharon durante los meses de junio, agosto y octubre del año 2013, en madurez fisiológica, de acuerdo con los parámetros visuales comerciales que se usan localmente, como son el tamaño, llenado del fruto, uniformidad e intensidad de color característico para cada cultivar, así como la ausencia de defectos y pudriciones. El material vegetal fue proveniente del Depositario Nacional de Opuntia, ubicado en las instalaciones del Centro Regional Universitario Centro Norte de la Universidad Autónoma Chapingo (UACh), en el municipio de El Orito, Zacatecas, México $\left(22^{\circ} 74^{\prime} 6^{\prime \prime} \mathrm{N}\right.$ y $\left.102^{\circ} 6^{\prime} 07^{\prime \prime} \mathrm{W}\right)$. El material se trasladó al Laboratorio de Usos Múltiples del Departamento de Fitotecnia de la Universidad Autónoma Chapingo, donde se realizaron todas las evaluaciones.

El diseño experimental fue completamente al azar, con cuatro repeticiones. Se consideró como unidad experimental un fruto. La evaluación fue por un periodo de 24 días y los frutos permanecieron a temperatura ambiente $\left(20 \pm 2{ }^{\circ} \mathrm{C}\right)$ $\mathrm{y}$ las determinaciones se realizaron en intervalos de 3 días a partir del día cero. Es importante señalar que este periodo de evaluación estuvo sujeto a las condiciones de deterioro visual del material vegetal, por lo que algunas variedades al presentar un deterioro evidente, la evaluación de las diferentes variables concluyó antes de lo previsto, y en el caso particular del contenido de clorofila total y carotenos, está fecha coincidió con la posibilidad de detectar la presencia de clorofila y carotenos, debido al método para su 
determinación y la mayor acentuación en la coloración característica de la pulpa del fruto en cada cultivar.

\section{Variables evaluadas \\ Capacidad antioxidante}

Se determinó de acuerdo al método ABTS (2,2'azino-bis 3-etilbenzotiazolina-6-ácido sulfónico) propuesto por Rice-Evans et al. (1997) y modificado por Ozgen et al. (2006). Una solución de ABTS $(7 \mathrm{mM})$ con persulfato de potasio se guardó en oscuridad durante $24 \mathrm{~h}$ a temperatura ambiente; transcurrido ese tiempo la solución se diluyó con amortiguador acetato de sodio $(\mathrm{pH} 4,5)$ hasta obtener un valor de absorbancia de $0,7 \pm 0,1$ a 734 nm (longitud de máxima absorción). El ensayo se realizó en las muestras de pulpa con 3,9 mL de solución ABTS y $100 \mu \mathrm{L}$ de extracto etanólico $(0,1 \mathrm{~g}$ de muestra fresca en $5 \mathrm{~mL}$ de etanol, homogeneizados con $24 \mathrm{~h}$ de reposo), después de un periodo de $2 \mathrm{~h}$ se realizó la lectura a $734 \mathrm{~nm}$. Para la cuantificación se obtuvo una curva patrón con ácido ascórbico y los valores se reportaron como actividad antioxidante equivalente a vitamina $\mathrm{C}$ (VCEAC) en $\mathrm{mg} \mathrm{g}^{-1}$ de peso fresco.

\section{Determinación de fenoles totales}

Se realizó a partir del extracto etanólico de pulpa obtenido para determinar la capacidad antioxidante, mediante el método de Folin y Ciocalteu descrito por Waterman y Mole (1994). A $300 \mu \mathrm{L}$ del extracto se le adicionaron $7,7 \mathrm{~mL}$ de agua desionizada, $0,5 \mathrm{~mL}$ del reactivo de Folin y Ciocalteu; la mezcla fue agitada por $10 \mathrm{~s}$ y se agregaron $1,5 \mathrm{~mL}$ de solución de carbonato de sodio al $20 \%$, posteriormente se dejó reposar durante 2 h en oscuridad y se procedió a tomar la lectura de absorbancia a $760 \mathrm{~nm}$. Los resultados se expresaron en equivalente de ácido gálico (EAG) en $\mathrm{mg} 100 \mathrm{~g}^{-1}$ de peso fresco, tomando como referencia una curva estándar de ácido gálico.

\section{Determinación de ácido ascórbico}

Se realizó mediante el método descrito por Jagota y Dani (1982). A 0,5 g de muestra de pulpa se le agregaron $5 \mathrm{~mL}$ de ácido tricloroacético al $10 \%$, posteriormente fue homogeneizada y agitada vigorosamente en un baño de hielo durante $5 \mathrm{~min}$, y se centrifugó a 22000 × g x 20 min. a $4^{\circ} \mathrm{C}$. De la mezcla obtenida se tomaron $200 \mu \mathrm{L}$ a los cuales se les agregaron 1,8 mL de agua bidestilada y $200 \mu \mathrm{L}$ del reactivo Folin y Ciocalteu en proporción de 1:10; la mezcla se dejó reposar durante $10 \mathrm{~min}$ y se obtuvo el valor de absorbancia de $760 \mathrm{~nm}$. El contenido de vitamina C se expresó en $\mathrm{mg} 100 \mathrm{~g}^{-1}$ de peso fresco mediante una curva estándar de ácido ascórbico.
Extracción y cuantificación de betalaína (betacianina y betaxantina).

La cuantificación de las betalaínas se realizó por espectrofotometría en las que se emplearon las ecuaciones de Nilsson (1970) y la metodología de Dantas et al. (2015). A 2 g de muestra de pulpa se le agregaron $10 \mathrm{~mL}$ de agua destilada, se homogeneizaron y posteriormente fueron centrifugados a 22000 × g x $25 \mathrm{~min}$. a $4^{\circ} \mathrm{C}$. De la solución que se obtuvo, se tomó el sobrenadante y se ajustó a un volumen de $30 \mathrm{~mL}$ con agua destilada, a los cuales se les determinó su valor de absorbancia en las longitudes de onda de 476, 538 y $600 \mathrm{~nm}$. Para el cálculo del contenido de betalaínas se utilizaron las siguientes ecuaciones: $x=1,095\left(A_{538}-A_{600}\right)$ y $y=-0,258 \times A_{538}+A_{476}-0,742$ x $A_{600}$. El contenido de betacianina se obtuvo por: $\mathrm{BTC}=(x \times R \times 100) / 1120, \mathrm{y}$ el de betaxantina por: $\mathrm{BTC}=(x \times R \times 100) / 750$, donde $R$ es el factor de dilución, y $E_{\mathrm{lcm}}^{1 \%}=1120$ y $E_{1 \mathrm{~cm}}^{1 \%}=750$, son los coeficientes de extinción para betanina e indicaxantina, respectivamente. Los resultados se expresaron como mg $100 \mathrm{~g}^{-1}$.

\section{Determinación de clorofila total y carotenos}

Se realizó mediante la técnica descrita por Lichtenthaler (1987). A 0,1 g de muestra de pulpa se le añadieron $5 \mathrm{~mL}$ de metanol al 100\% $\mathrm{y}$ fueron homogeneizados, posteriormente se centrifugaron a $22000 \times$ g x $25 \mathrm{~min}$. a $4^{\circ} \mathrm{C}$., y se obtuvo el valor de absorbancia a 665, 652 y 470 $\mathrm{nm}$. Los cálculos se realizaron con las fórmulas descritas a continuación: clorofila a: $C_{a}=16,72 A_{665,2}$ - 9,16 $A_{652,4} ;$ clorofila b: $C_{b}=34,09 A_{652,4}-15,28 A_{665,2} ;$ clorofila total: $C_{a+b}=1,44 A_{665,2}+24,93 A_{652,4} \mathrm{y}$ carotenos: $C_{x-c}=\left[1000 A_{470}-1,63 C_{a}-104,96 C_{b}\right] / 221$. Los resultados fueron expresados en $\mathrm{mg} 100 \mathrm{~g}^{-1}$ de peso fresco.

\section{Análisis estadístico}

Se realizó análisis de varianza (ANAVA) y una comparación de medias de Tukey $(\alpha=0,05)$, en las que se empleó el programa de análisis estadístico SAS ver. 9,0 (SAS, 2002).

\section{RESULTADOS Y DISCUSIÓN}

\section{Capacidad antioxidante}

La capacidad antioxidante fue mayor $(\alpha=$ 0,05 ) en los frutos provenientes de las diferentes variedades de xocotuna y xoconostle, cuyos valores fluctuaron entre 0,79 y $0,96 \mathrm{mg}$ VCEAC $\mathrm{g}^{-1}$ (Tabla 1) no obstante, cabe señalar que entre las variedades de tuna, se encontró que "Reyna" presentó un comportamiento similar a los materiales anteriormente mencionados. Los niveles encontrados de capacidad antioxidante coinciden con los reportados para frutos de 
Tabla 1. Capacidad antioxidante en frutos de cultivares de tuna, xocotuna y xoconostle, expresado como equivalente a vitamina $\mathrm{C}\left(\mathrm{mg} \mathrm{g}^{-1}\right)$.

Table 1. Antioxidant capacity in fruits of cactus pear, xocotuna and xoconostle cultivars, expressed as equivalent to vitamin $\mathrm{C}\left(\mathrm{mg} \mathrm{g}^{-1}\right)$.

\begin{tabular}{|c|c|c|c|c|c|c|c|c|c|}
\hline \multirow[t]{2}{*}{ Cultivar } & \multicolumn{8}{|c|}{ Días de evaluación } & \multirow[b]{2}{*}{24} \\
\hline & 0 & 3 & 6 & 9 & 12 & 15 & 18 & 21 & \\
\hline \multicolumn{10}{|l|}{ Tuna } \\
\hline 'Amarilla Montesa' (am) & $0,49 \mathrm{de}^{*}$ & $0,38 \mathrm{f}$ & $0,45 \mathrm{c}$ & $0,38 \mathrm{~g}$ & 0,43 ef & - & - & - & - \\
\hline ‘Amarilla Plátano' (am) & $0,42 \mathrm{e}$ & $0,37 \mathrm{f}$ & $0,45 c$ & $0,35 \mathrm{~g}$ & $0,38 \mathrm{f}$ & $0,40 \mathrm{c}$ & - & - & - \\
\hline ‘Reyna' (vc) & $0,79 \mathrm{abc}$ & $0,91 \mathrm{ab}$ & $0,76 a b$ & $0,80 \mathrm{bc}$ & $0,79 \mathrm{bc}$ & $0,89 \mathrm{a}$ & - & - & - \\
\hline 'Cristalina' (vc) & $0,37 \mathrm{e}$ & $0,38 \mathrm{f}$ & $0,37 \mathrm{c}$ & $0,39 \mathrm{fg}$ & $0,39 \mathrm{f}$ & - & - & - & - \\
\hline ‘Rojo Pelón’ (rj) & $0,51 \mathrm{de}$ & 0,52 ef & $0,50 \mathrm{c}$ & $0,44 \mathrm{efg}$ & 0,44 ef & $0,44 \mathrm{c}$ & $0,46 b$ & $0,51 b$ & $0,53 \mathrm{~b}$ \\
\hline 'Liso Forrajero' (rj) & $0,42 \mathrm{e}$ & $0,42 \mathrm{ef}$ & $0,42 \mathrm{c}$ & $0,39 \mathrm{~g}$ & $0,39 \mathrm{f}$ & $0,39 c$ & - & - & - \\
\hline \multicolumn{10}{|l|}{ Xocotuna } \\
\hline ‘Cascarón’ (rj) & $0,85 a b$ & 0,65 cde & $0,72 a b$ & 0,58 def & 0,62 cde & $0,71 \mathrm{abc}$ & $0,64 \mathrm{ab}$ & - & - \\
\hline 'Chinchilla' (rj) & 0,53 de & 0,61 def & $0,56 \mathrm{bc}$ & 0,62 cde & 0,51 def & - & - & - & - \\
\hline ‘Coral' (pu) & $0,88 \mathrm{ab}$ & $1,04 \mathrm{a}$ & $0,92 \mathrm{a}$ & $1,02 \mathrm{a}$ & 1,03 a & - & - & - & - \\
\hline 'Nicolaita' (rs) & 0,55 cde & 0,51 ef & $0,48 c$ & 0,48 defg & 0,64 cde & $0,50 \mathrm{bc}$ & - & - & - \\
\hline ‘Caidilla' (rj) & $0,67 \mathrm{bcd}$ & $0,77 \mathrm{bcd}$ & $0,76 a b$ & $0,67 \mathrm{~cd}$ & $0,69 \mathrm{bcd}$ & $0,82 \mathrm{ab}$ & - & - & - \\
\hline \multicolumn{10}{|l|}{ Xoconostle } \\
\hline 'Cuaresmeño Blanco' (vc) & $0,95 a$ & $0,84 \mathrm{abcd}$ & $0,76 a b$ & $0,77 \mathrm{bc}$ & $0,73 \mathrm{bc}$ & $0,77 \mathrm{ab}$ & $0,73 \mathrm{a}$ & 0,68 a & $0,76 \mathrm{a}$ \\
\hline ‘Cuaresmeño' (rj) & $0,96 \mathrm{a}$ & $0,89 \mathrm{abc}$ & $0,76 \mathrm{ab}$ & $0,90 \mathrm{ab}$ & $0,87 \mathrm{ab}$ & $0,80 \mathrm{ab}$ & $0,85 \mathrm{a}$ & $0,67 \mathrm{ab}$ & $0,86 \mathrm{a}$ \\
\hline DMSH & 0,24 & 0,25 & 0,21 & 0,18 & 0,22 & 0,31 & 0,21 & 0,16 & 0,17 \\
\hline
\end{tabular}

*Medias con igual letra dentro de la misma columna son estadísticamente iguales según la prueba de Tukey $(\alpha=0,05)$. am: amarillo; vc: verde claro; rj rojo; pu: púrpura; rs: rosa.

DMSH: diferencia mínima significativa honesta.

Opuntia ficus-indica y O. megacantha con valores

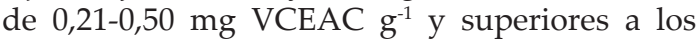
reportados para estas mismas especies, pero con valores de 0,13-0,30 $\mathrm{mg}$ VCEA g-1 $^{-1}$ y de 0,17 $0,33 \mathrm{mg}$ VCEAC $\mathrm{g}^{-1}$, respectivamente, después de 8 semanas de evaluación (Coria-Cayupán et al., 2011; Coria-Cayupán y Nazareno, 2015). Se ha señalado que las diferentes especies de frutos de Opuntia tienen capacidad antioxidante moderada, lo cual, podría estar directamente relacionado con el contenido de pigmentos de la fruta, o bien podría deberse al efecto del genotipo, tanto de especies como cultivares, así como a las condiciones de cultivo; asimismo, los valores encontrados en este estudio fueron semejantes a los encontrados para cítricos y otras frutas de temporada (Scalzo et al., 2005; Nazareno et al., 2008). Dadas las características de estos frutos, podrían ser considerados una fuente potencial de ingredientes funcionales que se pueden utilizar como aditivo en otros productos alimenticios (Figueroa-Cares et al., 2010; Hernández-Fuentes et al., 2015).

\section{Fenoles totales}

Se observó que a los 0, 3 y 6 días 'Cuaresmeño' fue estadísticamente diferente del resto de los cultivares, y presentó el valor inicial más alto con
44,61 mg EAG $100 \mathrm{~g}^{-1}$, seguido de 'Cuaresmeño Blanco' que tuvo diferencias significativas a los 3, 15 y 21 días. En contraste, 'Amarilla Plátano' y 'Liso Forrajero' presentaron las concentraciones más bajas con valores entre 6,36 y 5,13 mg EAG $100 \mathrm{~g}^{-1}$ (Tabla 2). En general, durante el periodo de evaluación se observó una disminución en la concentración de fenoles totales en la mayoría de los cultivares, lo que sugiere puede estar relacionado con las condiciones ambientales (temperatura, humedad relativa y luz), así como al manejo pre y poscosecha, donde se pueden incluir la nutrición del cultivo, el índice de cosecha y la metodología utilizada en la extracción de las muestras analizadas, tal y como lo señalaron Ramírez-Ramos et al. (2015). El comportamiento de esta variable contrasta con lo encontrado por Nazareno et al. (2008) para Opuntia spp., Opuntia ficus-indica y $O$. megacantha entre 2,23 y $73 \mathrm{mg}$ EAG $100 \mathrm{~g} \mathrm{~g}^{-1}$. Otros autores como Coria-Cayupán y Nazareno, (2015), reportan niveles muy superiores en accesiones de xoconostles (Opuntia spp.) que fueron de 108-313 mg EAG $100 \mathrm{~g}^{-1}$, todos estos resultados fueron determinados en la pulpa del fruto. En este sentido, se reconoce el efecto protector que los compuestos fenólicos poseen en contra del daño provocado por la luz ultravioleta y adicionalmente como un sistema de defensa 
Tabla 2. Contenido de fenoles totales (mg EAG $100 \mathrm{~g}^{-1}$ ) en frutos de cultivares de tuna, xocotuna y xoconostle (am: amarillo; vc: verde claro; rj rojo; pu: púrpura; rs: rosa).

Table 2. Total phenolic content (mg GAE $100 \mathrm{~g}^{-1}$ ) in fruits of cactus pear, xocotuna and xoconostle cultivars (am: yellow; vc: light green; rj: red; pu: purple; rs: pink).

\begin{tabular}{|c|c|c|c|c|c|c|c|c|c|}
\hline \multirow[t]{2}{*}{ Cultivar } & \multicolumn{9}{|c|}{ Días de evaluación } \\
\hline & 0 & 3 & 6 & 9 & 12 & 15 & 18 & 21 & 24 \\
\hline \multicolumn{10}{|l|}{ Tuna } \\
\hline 'Amarilla Montesa' (am) & $9,71 \mathrm{de}^{*}$ & $6,06 \mathrm{e}$ & 7,40 ef & 6,06 cde & $6,49 \mathrm{~d}$ & - & - & - & - \\
\hline 'Amarilla Plátano' (am) & $6,27 \mathrm{e}$ & $5,60 \mathrm{e}$ & $5,31 \mathrm{f}$ & 5,66 de & $5,71 \mathrm{~d}$ & $5,13 \mathrm{e}$ & - & - & - \\
\hline 'Reyna' (vc) & $19,56 \mathrm{bcd}$ & $21,21 \mathrm{c}$ & $17,35 \mathrm{~b}$ & $18,52 \mathrm{~b}$ & $16,65 b$ & $19,92 \mathrm{~b}$ & - & - & - \\
\hline 'Cristalina' (vc) & 17,56 de & $15,03 \mathrm{~cd}$ & $15,98 \mathrm{bcd}$ & $13,30 \mathrm{bc}$ & $15,99 \mathrm{~b}$ & - & - & - & - \\
\hline ‘Rojo Pelón' (rj) & $30,32 \mathrm{~b}$ & $15,76 \mathrm{~cd}$ & $16,07 \mathrm{bc}$ & $12,50 \mathrm{bcd}$ & $14,27 \mathrm{bc}$ & $13,73 \mathrm{c}$ & $13,65 b$ & $18,21 b$ & $17,81 \mathrm{~b}$ \\
\hline 'Liso Forrajero' (rj) & $6,36 \mathrm{e}$ & $5,80 \mathrm{e}$ & $5,67 \mathrm{f}$ & $4,68 \mathrm{e}$ & $4,77 \mathrm{~d}$ & $5,11 \mathrm{e}$ & - & - & - \\
\hline \multicolumn{10}{|l|}{ Xocotuna } \\
\hline 'Cascarón' (rj) & 11,91 de & $7,59 \mathrm{e}$ & 9,16 ef & 6,68 cde & $11,22 \mathrm{bcd}$ & 8,32 cde & $7,27 \mathrm{~b}$ & - & - \\
\hline 'Chinchilla' (rj) & 11,76 de & 9,28 de & 9,20 def & 9,93 cde & $7,37 \mathrm{~cd}$ & - & - & - & - \\
\hline ‘Coral' (pu) & $9,41 \mathrm{de}$ & 11,62 de & 12,95 bcde & 8,39 cde & $11,11 \mathrm{bcd}$ & $1-$ & - & - & - \\
\hline 'Nicolaita' (rs) & 7,07 de & $6,49 \mathrm{e}$ & $4,89 \mathrm{f}$ & 5,75 de & $5,82 \mathrm{~d}$ & 6,14 de & - & - & - \\
\hline 'Caidilla' (rj) & 10,30 de & 9,38 de & 10,16 cdef & 11,55 bcde & $9,30 \mathrm{bcd}$ & $11,35 \mathrm{~cd}$ & - & - & - \\
\hline \multicolumn{10}{|c|}{ Xoconostle } \\
\hline 'Cuaresmeño Blanco' (vc) & $29,61 b c$ & 52,31 a & $18,38 \mathrm{~b}$ & 28,46 a & 24,39 a & $33,62 \mathrm{a}$ & 28,86 a & $30,90 \mathrm{a}$ & $31,00 \mathrm{a}$ \\
\hline 'Cuaresmeño' (rj) & $44,61 \mathrm{a}$ & $30,19 b$ & $28,01 \mathrm{a}$ & $27,29 \mathrm{a}$ & 31,93 a & $23,24 b$ & $25,61 \mathrm{a}$ & $22,98 \mathrm{~b}$ & $28,13 \mathrm{a}$ \\
\hline DMSH & 12,58 & 6,92 & 6,79 & 7,50 & 7,57 & 5,48 & 7,29 & 4,82 & 9,79 \\
\hline
\end{tabular}

*Medias con igual letra dentro de la misma columna son estadísticamente iguales según la prueba de Tukey $(\alpha=0,05)$. DMSH: diferencia mínima significativa honesta.

frente al ataque de microorganismos patógenos, lo que podría hacer previsible el alto contenido de fenoles en la cáscara con relación a la pulpa; sin embargo, también existe la consideración de que dicha variabilidad podría estar asociada a factores como el tipo de cultivar, grado de madurez en la cosecha, condiciones climáticas y a la composición del suelo (Coria-Cayupán y Nazareno, 2015; Ramírez-Ramos et al., 2015).

\section{Contenido de ácido ascórbico}

El mayor contenido de vitamina $C$ se presentó en los cultivares de xoconostle ('Cuaresmeño blanco' y 'Cuaresmeño') de pulpa verde claro y roja, respectivamente; cuyos valores fluctuaron entre 41,80 y 137,61 mg $100 \mathrm{~g}^{-1}$ (Tabla 3), donde el valor más alto se presentó a los 18 días de evaluación en la variedad 'Cuaresmeño blanco'. Los resultados obtenidos de ácido ascórbico se encuentran dentro del intervalo reportados para Opuntia spp., O. ficus-indica y O. dillenii con valores entre 5,31 y 29,7 mg $100 \mathrm{~g}^{-1}$ (Figueroa-Cares et al., 2010; Díaz-Medina et al., 2007). Sin embargo, para xoconostle estos valores fueron superiores a lo encontrado por Guzmán-Maldonado et al. (2010) y por Gallegos-Vázquez et al. (2014) con contenidos de $38,1 \mathrm{mg} 100 \mathrm{~g}^{-1}$ para 'Cuaresmeño' y de $42,49 \mathrm{mg} 100 \mathrm{~g}^{-1}$ para 'Invierno', lo cual es importante debido al potencial que tienen estos compuestos en la disminución de la incidencia de estrés oxidativo y a la posterior aparición de enfermedades crónico-degenerativas (Frankel y German, 2006). La amplia variabilidad que se presentó entre especies y cultivares puede estar relacionada con la ruta de síntesis del ácido ascórbico, la cual difiere ligeramente en su eficiencia entre cultivares, de la misma forma en que influyen factores como las condiciones climáticas y edafológicas, adicionalmente a las condiciones de desarrollo del cultivo, puesto que el género Opuntia al estar adaptado a condiciones limitantes de suelo y agua, podría modificar su composición, principalmente la del fruto (Figueroa-Cares et al., 2010; Chougui et al., 2013).

\section{Betacianinas}

La concentración de betanina (rojo-violeta) fue mayor en las xocotunas, de tal manera que 'Coral' de pulpa púrpura y 'Caidilla' de pulpa roja fueron similares y significativamente diferentes del resto de los cultivares, al presentar los niveles más altos con valores de 70,08-84,41 mg $100 \mathrm{~g} \mathrm{~g}^{-1}$ a los 12 días y de 83,52-65,68 mg 100 $\mathrm{g}^{-1}$ a los 15 días, respetivamente. Los contenidos más bajos se observaron en 'Reyna' y 'Cristalina' de pulpa verde claro (entre 0,04 y 0,15 mg 100 
Tabla 3. Contenido de vitamina $\mathrm{C}\left(\mathrm{mg} 100 \mathrm{~g}^{-1}\right)$ en frutos de cultivares de tuna, xocotuna y xoconostle (am: amarillo; vc: verde claro; rj: rojo; pu: púrpura; rs: rosa).

Table 3. Content of vitamin $\mathrm{C}\left(\mathrm{mg}^{\left.100 \mathrm{~g} \mathrm{~g}^{-1}\right)}\right.$ in fruits of cactus pear, xocotuna and xoconostle cultivars (am: yellow; vc: light green; rj: red; pu: purple; rs: pink).

\begin{tabular}{|c|c|c|c|c|c|c|c|c|c|}
\hline \multirow[t]{2}{*}{ Cultivar } & \multicolumn{9}{|c|}{ Días de evaluación } \\
\hline & 0 & 3 & 6 & 9 & 12 & 15 & 18 & 21 & 24 \\
\hline \multicolumn{10}{|l|}{ Tuna } \\
\hline 'Amarilla Montesa' (am) & $5,92 \mathrm{f}^{*}$ & $5,45 \mathrm{e}$ & $5,52 \mathrm{~d}$ & $3,58 \mathrm{c}$ & $2,34 \mathrm{f}$ & - & - & - & - \\
\hline 'Amarilla Plátano' (am) & 21,95 def & 24,27 de & $19,20 \mathrm{~cd}$ & $25,28 c$ & 20,96 def & $23,06 \mathrm{~d}$ & - & - & - \\
\hline ‘Reyna' (vc) & 16,90 def & 21,43 de & $15,41 \mathrm{~cd}$ & $20,34 \mathrm{c}$ & 23,83 def & $21,83 \mathrm{~d}$ & - & - & - \\
\hline 'Cristalina' (vc) & 13,26 ef & 14,46 e & $13,31 \mathrm{~cd}$ & $14,16 \mathrm{c}$ & 17,26 ef & - & - & - & - \\
\hline ‘Rojo Pelón' (rj) & $37,53 \mathrm{~cd}$ & 28,12 de & $33,96 \mathrm{bcd}$ & $25,76 \mathrm{c}$ & 25,87 de & $29,31 \mathrm{~d}$ & $29,81 \mathrm{c}$ & $42,54 b$ & $38,98 \mathrm{c}$ \\
\hline 'Liso Forrajero' (rj) & 19,70 def & $16,00 \mathrm{e}$ & $19,46 \mathrm{~cd}$ & $21,61 \mathrm{c}$ & 16,99 ef & $24,74 \mathrm{~d}$ & - & - & - \\
\hline \multicolumn{10}{|l|}{ Xocotuna } \\
\hline ‘Cascarón’ (rj) & 32,34 cde & 25,68 de & $30,07 \mathrm{bcd}$ & $26,22 \mathrm{c}$ & 28,87 de & $31,21 \mathrm{~d}$ & $39,64 \mathrm{c}$ & - & - \\
\hline 'Chinchilla' (rj) & $9,64 \mathrm{f}$ & 8,03 e & $10,53 \mathrm{~d}$ & $10,73 \mathrm{c}$ & $10,90 \mathrm{ef}$ & - & - & - & - \\
\hline 'Coral' (pu) & $49,75 \mathrm{bc}$ & $59,74 \mathrm{bc}$ & $43,26 \mathrm{bc}$ & $59,87 \mathrm{~b}$ & $68,03 \mathrm{~b}$ & - & - & - & - \\
\hline 'Nicolaita' (rs) & $12,13 \mathrm{ef}$ & 18,99 de & $12,52 \mathrm{~cd}$ & $11,43 \mathrm{c}$ & 16,56 ef & $13,93 \mathrm{~d}$ & - & - & - \\
\hline 'Caidilla' (rj) & $63,97 \mathrm{~b}$ & $42,21 \mathrm{~cd}$ & $51,27 \mathrm{~b}$ & $58,01 \mathrm{~b}$ & $51,83 \mathrm{bc}$ & $56,66 \mathrm{~d}$ & - & - & - \\
\hline \multicolumn{10}{|c|}{ Xoconostle } \\
\hline 'Cuaresmeño Blanco' (vc) & ) $95,99 a$ & $79,16 \mathrm{ab}$ & $96,01 \mathrm{a}$ & $92,25 \mathrm{a}$ & $41,80 \mathrm{~cd}$ & $94,04 \mathrm{~b}$ & $137,61 \mathrm{a}$ & $79,29 \mathrm{ab}$ & $125,98 \mathrm{a}$ \\
\hline 'Cuaresmeño' (rj) & $103,72 \mathrm{a}$ & 97,40 a & 109,65 a & 96,67 a & 95,71 a & 125,22 a & $97,99 \mathrm{~b}$ & 110,41 a & $70,39 \mathrm{~b}$ \\
\hline DMSH & 22,04 & 26,07 & 31,08 & 28,11 & 22,17 & 24,84 & 21,75 & 39,17 & 27,11 \\
\hline
\end{tabular}

*Medias con igual letra dentro de la misma columna son estadísticamente iguales según la prueba de Tukey $(\alpha=0,05)$. DMSH: diferencia mínima significativa honesta.

$\left.\mathrm{g}^{-1}\right)$, mientras que en 'Cuaresmeño Blanco' no se detectó este pigmento. Asimismo, se observó una disminución de este pigmento en la mayoría de los cultivares evaluados al pasar los días de evaluación, asimismo el contenido de este pigmento fue mayor en los frutos de xocotuna (Tabla 4). Respecto al contenido de betacianinas, los resultados obtenidos fueron similares a lo reportado para Opuntia sp., Opuntia ficus-indica, O. streptacantha, O. pumila, O. stricta, Opuntia robusta, Opuntia matudae entre 0,16 y 86,69 mg $100 \mathrm{~g}^{-1}$ (Dantas et al., 2015; Guzmán-Maldonado et al., 2010; Chavéz-Santoscoy et al., 2009; Aquino et al., 2012). Las determinaciones cuantitativas permitieron observar la presencia de diferentes colores en los frutos, que están relacionados con las variaciones en el contenido de betalaínas, el cual también es afectado por factores como el cultivar, la distribución en el fruto (cáscara o pulpa), y el clima o sitio geográfico de distribución. Aquellos frutos que presentan mayor contenido podrían ser utilizados como fuente de ingrediente funcional para la industria alimenticia y tener una aplicación potencial como colorante natural (Saénz et al., 2012).

\section{Indicaxantina}

Con respecto al contenido de betaxantina (amarillo-naranja), destacó 'Caidilla' de pulpa roja con valores que fueron de 54,68-54,10 mg $100 \mathrm{~g}^{-1}$, seguida de 'Amarilla Plátano' que fue de 47,88-45,02 mg $100 \mathrm{~g}^{-1}$ a los 15 días y 'Amarilla Montesa' con niveles de 30,77-109,46 mg $100 \mathrm{~g}^{-1}$ a los 12 días, estas dos últimas de pulpa amarilla. Se encontró en menor concentración en 'Reyna', 'Cristalina' y 'Cuaresmeño' de pulpa verde claro con valores entre 0,19 y 2,35 mg $100 \mathrm{~g}^{-1}$, asimismo en 'Cuaresmeño Blanco' no fue detectado dicho pigmento. Este pigmento se fue incrementando en la mayoría de los cultivares durante los días de evaluación (Tabla 5). La concentración de betaxantina contrasta con lo señalado por Chavéz-Santoscoy et al. (2009) para cultivares de Opuntia spp., con valores entre 0,31 y 18,99 mg $100 \mathrm{~g}^{-1}$ y lo indicado por Coria-Cayupán et al. (2011) y Dantas et al. (2015) para Opuntia stricta, O. megacantha y O. ficus-indica entre 1,01 y 10,33 mg $100 \mathrm{~g}^{-1}$. En relación con este aspecto, Guzmán-Maldonado et al. (2010) indican que la variación del color de la cascara del fruto, así como la considerable diversidad de coloraciones 
Tabla 4. Contenido de betanina ( $\mathrm{mg} 100 \mathrm{~g}^{-1}$ ) en frutos de cultivares de tuna, xocotuna y xoconostle (am: amarillo; vc: verde claro; rj: rojo; pu: púrpura; rs: rosa).

Table 4. Betanin content ( $\mathrm{mg} 100 \mathrm{~g}^{-1}$ ) in fruits of cactus pear, xocotuna and xoconostle cultivars (am: yellow; vc: light green; rj: red; pu: purple; rs: pink).

\begin{tabular}{|c|c|c|c|c|c|c|c|c|c|}
\hline \multirow[t]{2}{*}{ Cultivar } & \multicolumn{9}{|c|}{ Días de evaluación } \\
\hline & 0 & 3 & 6 & 9 & 12 & 15 & 18 & 21 & 24 \\
\hline \multicolumn{10}{|l|}{ Tuna } \\
\hline 'Amarilla Montesa' (am) & $0,97 d^{*}$ & $1,29 \mathrm{~d}$ & $0,99 \mathrm{ef}$ & $0,94 \mathrm{~d}$ & $3,07 \mathrm{~d}$ & - & - & - & - \\
\hline ‘Amarilla Plátano' (am) & $0,43 \mathrm{~d}$ & $0,40 \mathrm{~d}$ & $0,34 \mathrm{f}$ & $0,27 \mathrm{~d}$ & $0,31 \mathrm{~d}$ & 0,37 e & - & - & - \\
\hline ‘Reyna' (vc) & $0,04 \mathrm{~d}$ & $0,04 \mathrm{~d}$ & $0,11 \mathrm{f}$ & $0,15 \mathrm{~d}$ & $0,05 \mathrm{~d}$ & $0,11 \mathrm{e}$ & - & - & - \\
\hline 'Cristalina' (vc) & $0,05 \mathrm{~d}$ & $0,06 \mathrm{~d}$ & $0,04 \mathrm{f}$ & $0,09 \mathrm{~d}$ & $0,08 \mathrm{~d}$ & - & - & - & - \\
\hline ‘Rojo Pelón’ (rj) & $12,66 \mathrm{~cd}$ & $14,62 \mathrm{~cd}$ & 17,93 de & $15,61 \mathrm{c}$ & $16,77 \mathrm{~cd}$ & $17,44 \mathrm{~cd}$ & $16,70 \mathrm{~b}$ & $19,11 \mathrm{a}$ & 22,25 \\
\hline 'Liso Forrajero' (rj) & $7,97 \mathrm{~cd}$ & $5,58 \mathrm{~d}$ & 7,75 def & $11,82 \mathrm{~cd}$ & $6,98 \mathrm{~cd}$ & 7,41 de & - & - & - \\
\hline \multicolumn{10}{|l|}{ Xocotuna } \\
\hline 'Cascarón' (rj) & $44,62 \mathrm{~b}$ & $49,09 \mathrm{~b}$ & $40,79 \mathrm{~b}$ & $37,70 \mathrm{~b}$ & $46,45 \mathrm{~b}$ & $38,98 \mathrm{~b}$ & $36,29 a$ & - & - \\
\hline 'Chinchilla' (rj) & $33,10 \mathrm{~b}$ & $26,27 \mathrm{c}$ & $37,24 \mathrm{bc}$ & $29,90 \mathrm{~b}$ & $30,13 \mathrm{bc}$ & - & - & - & - \\
\hline ‘Coral' (pu) & 70,08 a & 94,68 a & $79,30 \mathrm{a}$ & 78,40 a & 84,41 a & - & - & - & - \\
\hline 'Nicolaita' (rs) & $19,38 \mathrm{c}$ & $15,23 \mathrm{~cd}$ & $21,05 \mathrm{~cd}$ & $16,25 c$ & $18,86 \mathrm{~cd}$ & $19,26 \mathrm{c}$ & - & - & - \\
\hline 'Caidilla' (rj) & $83,52 \mathrm{a}$ & 81,26 a & 84,79 a & $83,70 \mathrm{a}$ & $73,04 \mathrm{a}$ & $65,68 \mathrm{a}$ & - & - & - \\
\hline \multicolumn{10}{|l|}{ Xoconostle } \\
\hline 'Cuaresmeño Blanco' (vc) & ND & ND & ND & ND & ND & ND & ND & ND & ND \\
\hline 'Cuaresmeño' (rj) & $1,18 \mathrm{~d}$ & $1,10 \mathrm{~d}$ & 0,89 ef & $1,31 \mathrm{~d}$ & $1,05 \mathrm{~d}$ & $1,57 \mathrm{e}$ & $2,20 c$ & $0,80 \mathrm{~b}$ & $0,97 \mathrm{~b}$ \\
\hline DMSH & 13,67 & 15,89 & 17,11 & 11,75 & 26,13 & 10,5 & 6,93 & 2,33 & 3,60 \\
\hline
\end{tabular}

*Medias con igual letra dentro de la misma columna son estadísticamente iguales según la prueba de Tukey $(\alpha=0,05)$.

DMSH: diferencia mínima significativa honesta; ND: no detectado.

Tabla 5. Contenido de indicaxantina ( $\left.\mathrm{mg} 100 \mathrm{~g} \mathrm{~g}^{-1}\right)$ en frutos de cultivares de tuna, xocotuna y xoconostle (am: amarillo; vc: verde claro; rj: rojo; pu: púrpura; rs: rosa).

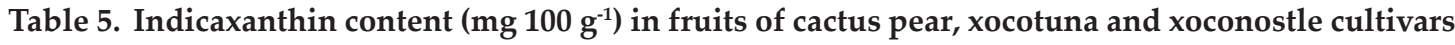
(am: yellow; vc: light green; rj: red; pu: purple; rs: pink).

\begin{tabular}{|c|c|c|c|c|c|c|c|c|c|}
\hline \multirow[t]{2}{*}{ Cultivar } & \multicolumn{7}{|c|}{ Días de evaluación } & \multirow[b]{2}{*}{21} & \multirow[b]{2}{*}{24} \\
\hline & 0 & 3 & 6 & 9 & 12 & 15 & 18 & & \\
\hline \multicolumn{10}{|l|}{ Tuna } \\
\hline 'Amarilla Montesa' (am) & $30,77 b^{*}$ & $35,93 \mathrm{bc}$ & $38,84 \mathrm{~b}$ & $42,64 \mathrm{~b}$ & 109,46 a & - & - & - & - \\
\hline 'Amarilla Plátano' (am) & 47,88 a & $30,73 \mathrm{bc}$ & $33,96 \mathrm{bc}$ & $76,28 \mathrm{a}$ & $44,75 \mathrm{bc}$ & $45,02 \mathrm{a}$ & - & - & - \\
\hline 'Reyna' (vc) & $0,21 \mathrm{~d}$ & 0,24 e & $0,48 \mathrm{e}$ & $0,41 \mathrm{~d}$ & $0,25 \mathrm{e}$ & $0,37 \mathrm{c}$ & - & - & - \\
\hline 'Cristalina' (vc) & $0,19 \mathrm{~d}$ & $0,35 \mathrm{e}$ & $0,21 \mathrm{e}$ & $0,34 \mathrm{~d}$ & $0,42 \mathrm{e}$ & - & - & - & - \\
\hline ‘Rojo Pelón’ (rj) & $9,52 \mathrm{~cd}$ & 13,62 de & $13,36 \mathrm{de}$ & $14,14 \mathrm{~cd}$ & 18,12 de & $21,78 \mathrm{~b}$ & $11,60 \mathrm{~b}$ & $11,23 \mathrm{a}$ & $19,91 \mathrm{a}$ \\
\hline 'Liso Forrajero' (rj) & $8,85 \mathrm{~d}$ & 9,21 e & $8,59 \mathrm{e}$ & $9,87 \mathrm{~d}$ & $4,92 \mathrm{e}$ & $7,53 \mathrm{bc}$ & - & - & - \\
\hline \multicolumn{10}{|l|}{ Xocotuna } \\
\hline ‘Cascarón' (rj) & $16,07 \mathrm{bcd}$ & $25,72 \mathrm{~cd}$ & $24,41 \mathrm{~cd}$ & $25,35 \mathrm{bcd}$ & $27,97 \mathrm{~cd}$ & $23,06 \mathrm{~b}$ & 29,21 a & - & - \\
\hline ‘Chinchilla' (rj) & $9,91 \mathrm{~cd}$ & $10,06 \mathrm{e}$ & 12,79 de & $18,13 \mathrm{bcd}$ & 14,81 de & - & - & - & - \\
\hline 'Coral' (pu) & $25,63 \mathrm{bc}$ & $42,26 b$ & $34,10 \mathrm{bc}$ & $42,25 \mathrm{bc}$ & $49,30 \mathrm{~b}$ & - & - & - & - \\
\hline 'Nicolaita' (rs) & $9,07 \mathrm{~d}$ & $7,42 \mathrm{e}$ & 13,21 de & $8,34 \mathrm{~d}$ & $7,90 \mathrm{e}$ & $9,87 \mathrm{bc}$ & - & - & - \\
\hline 'Caidilla' (rj) & 54,68 a & 82,38 a & $93,42 \mathrm{a}$ & 78,19 a & $55,16 \mathrm{~b}$ & 54,10 a & - & - & - \\
\hline \multicolumn{10}{|c|}{ Xoconostle } \\
\hline 'Cuaresmeño Blanco' (vc) & ND & ND & ND & ND & ND & ND & ND & ND & ND \\
\hline 'Cuaresmeño' (rj) & $0,34 \mathrm{~d}$ & 0,37 e & $0,30 \mathrm{e}$ & $0,77 \mathrm{~d}$ & $0,52 \mathrm{e}$ & $2,35 \mathrm{bc}$ & $0,95 \mathrm{c}$ & $0,54 \mathrm{~b}$ & $0,44 \mathrm{~b}$ \\
\hline DMSH & 16,33 & 13,76 & 13,83 & 28,28 & 18,74 & 21,07 & 7,78 & 7,36 & 7,8 \\
\hline
\end{tabular}

*Medias con igual letra dentro de la misma columna son estadísticamente iguales según la prueba de Tukey $(\alpha=0,05)$. DMSH: diferencia mínima significativa honesta; ND: no detectado 
en el mucílago que une y rodea a las semillas, se encuentra estrechamente relacionado con el contenido de betalainas.

\section{Clorofila total y carotenos}

El contenido de clorofila total se detectó solamente en 'Amarilla Montesa', 'Amarilla Plátano', 'Reyna', 'Cristalina' y 'Cuaresmeño Blanco', con pulpa de coloración amarilla o verde claro; en el resto de los cultivares no fue detectada debido a que presentaron pulpa de coloración roja, rosa o púrpura. En términos generales, el comportamiento de esta variable entre los cultivares de tuna ('Amarilla Montesa', 'Reyna' y 'Cristalina') y xoconostle ('Cuaresmeño Blanco') fue similar $(\alpha=0,05)$, excepto a los 6 y 9 días de evaluación, con valores de clorofila total entre 43,11 y 75,95 mg $100 \mathrm{~g}^{-1}$ (Tabla 6), donde en la mayoría de los casos se observó un incrementó en el contenido de este pigmento. En relación con esta variable, Figueroa-Cares et al. (2010) indicaron valores entre 118,82 y 125,59 mg 100 $\mathrm{g}^{-1}$ para frutos de tuna blanca y amarilla (Opuntia spp.) superiores a los encontrados en el presente estudio, lo que permite inferir que la relación entre el color de la pulpa y la presencia de clorofila es indirecta debido a las modificaciones en la composición de los pigmentos durante el periodo evaluado (Coria-Cayupán et al., 2011), por tanto esta característica es un mal indicador del contenido de pigmentos en estos frutos.
Asimismo, los carotenos se determinaron únicamente en 'Amarilla Montesa' y 'Amarilla Plátano' de pulpa amarilla, debido a que en los demás cultivares no fue detectado por presentar pulpa de coloración verde claro, roja, rosa o púrpura. A excepción del día 3 de evaluación, se encontró que el cultivar 'Amarilla Montesa' presentó la mayor concentración de carotenos con valores de $34,95-93,28 \mathrm{mg} 100 \mathrm{~g}^{-1}$, en la que se observó un incremento conforme avanzaron los días de evaluación (Tabla 6). Al respecto, se encontró que la concentración de carotenos fue superior a la indicada para Opuntia ficus-indica, Opuntia stricta y Opuntia undulata, con valores de 2,58, 4,71 y 6,68 mg $100 \mathrm{~g}^{-1}$, respectivamente, y menores a los mostrados para 'Naranjona' que fueron de $85-52 \mathrm{mg} 100 \mathrm{~g}^{-1}$ a los 14 días (Hernández-Pérez et al., 2005; Fernández-López et al., 2010). En este contexto, la variabilidad en el contenido de carotenos puede ser debida a varios factores, tales como el tipo de fruto, cultivar, las diferencias en los constituyentes del mismo y los métodos usados para su extracción y cuantificación (Hernández-Pérez et al., 2005; Corral-Aguayo et al., 2008).

\section{CONCLUSIONES}

Las variedades de xoconostle se destacaron por presentar el mayor nivel de capacidad antioxidante y fenoles totales; así como de

Tabla 6. Contenido de clorofila total y carotenos $\left(\mathrm{mg} 100 \mathrm{~g}^{-1}\right)$ en frutos de cultivares de tuna, xocotuna y xoconostle (am: amarillo; vc: verde claro; rj: rojo; pu: púrpura; rs: rosa).

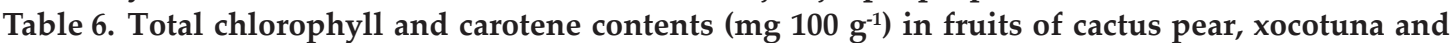
xoconostle cultivars (am: yellow; vc: light green; rj: red; pu: purple; rs: pink).

\begin{tabular}{|c|c|c|c|c|c|c|}
\hline \multirow[t]{2}{*}{ Cultivar } & \multicolumn{6}{|c|}{ Días de evaluación } \\
\hline & 0 & 3 & 6 & 9 & 12 & 15 \\
\hline \multicolumn{7}{|l|}{ CLOROFILA TOTAL } \\
\hline \multicolumn{7}{|l|}{ Tuna } \\
\hline 'Amarilla Montesa' (am) & $70,11 a^{*}$ & 71,67 a & $75,95 \mathrm{a}$ & 68,99 a & $70,75 \mathrm{a}$ & - \\
\hline 'Amarilla Plátano' (am) & $19,55 \mathrm{~b}$ & $17,79 \mathrm{~b}$ & $22,73 \mathrm{c}$ & $18,85 \mathrm{c}$ & $21,97 \mathrm{~b}$ & $25,56 \mathrm{~b}$ \\
\hline 'Reyna' (vc) & $47,15 \mathrm{a}$ & 48,18 a & $65,04 \mathrm{ab}$ & $44,88 \mathrm{~b}$ & $46,84 \mathrm{ab}$ & $43,80 \mathrm{ab}$ \\
\hline 'Cristalina' (vc) & $53,23 \mathrm{a}$ & $53,19 \mathrm{a}$ & $52,16 \mathrm{~b}$ & $59,75 \mathrm{ab}$ & $56,94 \mathrm{a}$ & - \\
\hline \multicolumn{7}{|l|}{ Xoconostle } \\
\hline 'Cuaresmeño Blanco' (vc) & 46,53 a & $43,11 \mathrm{ab}$ & $49,88 \mathrm{~b}$ & $53,85 \mathrm{ab}$ & 59,32 a & 53,14 a \\
\hline DMSH & 25,62 & 29,03 & 21,2 & 22,24 & 33,64 & 20,77 \\
\hline \multicolumn{7}{|l|}{ CAROTENOS } \\
\hline \multicolumn{7}{|l|}{ Tuna } \\
\hline 'Amarilla Montesa' (am) & $34,95 \mathrm{a}$ & $32,54 \mathrm{a}$ & 34,53 a & $46,81 \mathrm{a}$ & 93,28 a & - \\
\hline 'Amarilla Plátano' (am) & $27,81 \mathrm{~b}$ & $27,54 \mathrm{a}$ & $25,76 b$ & $36,67 \mathrm{~b}$ & $30,15 b$ & - \\
\hline DMSH & 3,17 & 8,97 & 6,30 & 9,23 & 11,69 & - \\
\hline
\end{tabular}

*Medias con igual letra dentro de la misma columna son estadísticamente iguales según la prueba de Tukey $(\alpha=0,05)$. DMSH: diferencia mínima significativa honesta. 
vitamina $C$, donde también se incluyen a tunas y xocotunas; por lo que podrían ser considerados como una excelente alternativa para ser incorporados a la dieta o como ingredientes naturales en la elaboración de otros productos. La concentración de pigmentos (betalaínas, clorofila total y carotenos) estuvo relacionada con el color característico del fruto de cada variedad.

\section{LITERATURA CITADA}

Aquino B.E.N., Y.C. Moctezuma, J. L. C. Servia, R. I. G. Gerónimo, E. R. S. Hernández y I. V. Guzmán. 2012. Caracterización fisicoquímica de siete variedades de tuna (Opuntia spp.) color rojo-violeta y estabilidad del pigmento de las dos variedades con mayor concentración. Investigación y Ciencia 55:3-10.

Bravo H., H. y L. Scheinvar. 1999. El interesante mundo de las cactáceas. 233 p. $2^{a}$ ed. Fondo de Cultura Económica, D. F., México.

Chavéz-Santoscoy, R.A., J. A. Gutiérrez-Uribe, and S.O. Serna-Saldívar. 2009. Phenolic composition, antioxidant capacity and in vitro cancer cell cytoxicity of nine prickly pear (Opuntia spp.) juices. Plant Foods for Human Nutrition 64:146-152.

Chougui, N., Louaileche, H. Mohedeb, S. Mouloudj, Y. Hammoui, and A. Tamendjari. 2013. Physico-chemical characterization and antioxidant activity of some Opuntia ficusindica varieties grown in North Algeria. African Journal of Biotechnology 12(3):299307.

Coria-Cayupán, Y., and M.A. Nazareno, 2015. Betalain profile and antioxidant phytochemicals of Opuntia fruits from Santiago del Estero, Argentina. Acta Horticulturae 1067:311-317.

Coria-Cayupán, Y., Ma. J. Ochoa, and M. A. Nazareno. 2011. Health-promoting substances and antioxidant properties of Opuntia sp. fruits. Changes in bioactivecompound contents during ripening process. Food Chemistry 126:514-519.

Corral-Aguayo, R.D., E.M. Yahia, A. CarrilloLópez, and G. González-Aguilar. 2008. Correlation between some nutritional components and the total antioxidant capacity measured with six different assays in eight horticultural crops. Journal Agricultural and Food Chemistry 56:1049810504.
Dantas, R.L., S.M. Silva, D.M. Brito Primo, A.S.B. Sousa, E.S. Brito, and E.M.S. Macedo. 2015. Changes during maturation in the bioactive compounds and antioxidant activity of Opuntia stricta (Haw.) fruits. Acta Horticulturae 1067:159-165.

Díaz-Medina, E. M., E. M. Rodríguez-Rodríguez, and C. Díaz-Romero. 2007. Chemical characterization of Opuntia dillenii and Opuntia ficus indica fruits. Food Chemistry 103: 38-45.

Fernández-López, J.A., L. Almela, J.M. Obón, and R. Castellar. 2010. Determination of antioxidant constituents in cactus pear fruits. Plant Foods for Human Nutrition 65:253-259.

Figueroa-Cares, I., M.T. Martínez-Damián, E. Rodríguez-Pérez, M.T. Colinas-León, S. Valle-Guadarrama, S. Ramírez-Ramírez, et al. 2010. Contenido de pigmentos, otros compuestos y capacidad antioxidante en 12 cultivares de tuna (Opuntia spp.) de México. Agrociencia 44(7):763-771.

Frankel, E.N., and J. B German. 2006. Antioxidants in foods and health: problems and fallacies in the field. Journal of the Science of Food and Agriculture 86:1999-2001.

Gallegos-Vázquez, C., A.F. Barrientos-Priego, J.A. Reyes-Agüero, C.A. Núñez-Colín, and C. Mondragón-Jacobo. 2011. Clusters of commercial varieties of cactus pear and xoconostle using UPOV morphological traits. Journal of the Professional Association for Cactus Development 13:10-22.

Gallegos-Vázquez, C., L. Scheinvar, H. SilosEspino, A.D. Fuentes-Hernández, C. A. Núñez-Colín, y G. Olalde-Parra. 2014. 'Invierno', cultivar de xoconostle para la región central de México. Revista Mexicana de Ciencias Agrícolas 7:1349-1354.

Guzmán-Maldonado, S.H., A.L MoralesMontelongo, C. Mondragón-Jacobo, G. Herrera-Hernández, F. Guevara-Lara, and R. Reynoso-Camacho. 2010. Physicochemical, nutritional, and functional characterization of fruits xoconostle (Opuntia matudae) pears from Central-México region. Journal of Food Science 75(6):485-492.

Hernández-Fuentes, A.D., A. Trapala-Islas, C. Gallegos-Vázquez, R.G. Campos-Montiel, J.M Pinedo-Espinoza, and S.H. GuzmánMaldonado. 2015. Physicochemical variability and nutritional and functional characteristics of xoconostles (Opuntia spp.) accessions from México. Fruits 70(2):109-116. 
Hernández-Pérez, T., A. Carrillo-López, F. Guevara-Lara, A. Cruz-Hernández, and O. Paredes-López. 2005. Biochemical and nutritional characterization of three prickly pear species with different ripening behavior. Plants Foods for Human Nutrition 60: 195-200.

Jagota, S.K., and H. M. Dani. 1982. A new colorimetric technique for the estimation of vitamin C using Folin phenol reagent. Analytical Biochemistry 127:178-182.

Lichtenthaler, H.K. 1987. Chloropphyls and carotenoids, pigments of photosynthetic biomembranes. p. 350-383. In L. Packer y R. Donce (eds.) Methods in enzymology. Academic Press Inc., New York, USA.

Nazareno, M.A., E. González, M. García, C. Loto, and Y. Coria-Cayupán. 2008. Actividad antioxidante de alimentos consumidos en Argentina. Industria Química 358:58-63.

Nilsson, T. 1970. Studies into the pigments in beetroot (Beta vulgaris L. ssp. vulgaris var. rubra L.). Lantbrukshögskolans Annaler 36:179-219.

Osorio-Esquivel, O., A. Ortíz-Moreno, V.B. Álvarez, L. Dorantes-Álvarez, and M.M. Giusti. 2011. Phenolics, betacyanins and antioxidant activity in Opuntia joconostle fruits. Food Research International 44:21602168.

Ozgen, M., R. Reese, R. Neil, Jr.A.Z. Tulio, J.C. Scheerens, and A.M. Raymond. 2006. Modified 2,2-azino-bis-3ethilbenzothiazoline-6-sulfonic acid (ABTS) method to measure antioxidant capacity of selected small fruits and comparison to ferric reducing antioxidant power (FRAP) and 2,2'-diphenyl-1-picrylhydrazyl (DPPH) Methods. Journal Agricultural and Food Chemistry 54:1151-1157.

Pimienta-Barrios, E., L. Méndez-Morán, J.E. Ramírez-Hernández, J.E. Alba-García, and R.M. Domínguez-Arias. 2008. Efecto sobre la ingestión del fruto de xoconostle (Opuntia joconostle Web.) sobre la glucosa y lípidos séricos. Agrociencia 42:645-653.
Prieto-García, F., M.A. Méndez-Marzo, E. Pérez-Cruz, S. Filardo-Kerstupp, and B.R. Cruz-Martínez. 2008. Morfología, tamaño y distribución de partículas en semillas de cinco especies del género Opuntia. Universidad y Ciencia Trópico Húmedo 24(2):159-162.

Ramírez-Ramos, M., Ma. R. García-Mateos, J. Corrales-García, C. Ybarra-Moncada, and A. Ma. Castillo-González. 2015. Compuestos antioxidantes en variedades pigmentadas de tuna (Opuntia sp.). Revista Fitotecnia Mexicana 38(4):349-357.

Reyes-Agüero, J.A., J.R.R. Aguirre, and J.J. F. Flores. 2005. Variación morfológica de Opuntia (cactaceae) en relación con su domesticación en la altiplanicie meridional de México. Interciencia 30(8):476-484.

Rice-Evans, A.C., N.J. Miller, and G. Paganga. 1997. Antioxidant properties of phenolic compounds. Trends in Plant Science 2(4):152158.

Saénz, C. 2006. Opuntia spp. Bioactive compounds in foods: a Plus for health. Acta Horticulturae 728:231-240.

Saénz, C., B. Cancino, and P. Robert. 2012. Red betalains from Opuntia spp.: Natural colorants with potential applications in foods. Israel Journal of Plant Sciences 60:291299.

SAS. 2002. SAS/STAT user's guide: Statistics. Ver. 9.00. 1503 p. SAS Institute Inc., Cary, North Caroline, USA.

Scalzo, J., A. Politi, N. Pellegrini, B. Mezzetti, and M. Battino. 2005. Plant genotype affects total antioxidant capacity and phenolic contents in fruit. Nutrition 21:207-213.

Scheinvar, L., and G. Manzanero. 2009. Opuntia chianguiana, Una nueva especie de Cactaceae de Oaxaca, México. Journal for Botanical Nomenclature 19(2):222-228.

Waterman, P.G., and S. Mole. 1994. Analysis of Phenolic Plant Metabolites. 238 p. Blackwell Scientific Publications, Oxford. UK. 